\title{
Behaviour of Secondary Coolant Flow on the Turbine Blade Surface
}

\author{
Bhaskar Roy, Preetam S \\ Aerospace Engineering Department, Indian Institute of Technology Bombay \\ I.I.T., Powai, Mumbai, India \\ aeroyia@aero.iitb.ac.in; 143010023@iitb.ac.in
}

\begin{abstract}
In this paper, the fluid mechanic behaviour of secondary flows, cooling injection from inside the blade, on the turbine blades surfaces has been studied for its aerodynamic influence. A standard T-106A turbine blade profile has been chosen for study. The blades are arranged in a linear cascade with a solidity of 0.5 and aspect ratio of 1.5 . It is first intended to find the most effective single blowing location, which has been found at $10 \%$ of the blade surface from the leading edge. Then the study has focussed on finding a blowing ratio at which the secondary flow boundary layer sustains itself over most part of the turbine blade surfaces - both the suction and the pressure surfaces. Further, fluid mechanic studies have been conducted at this effective blowing ratio $(\approx 0.8)$ to explore the boundary layer behaviour of the injected cooling flow. The boundary layer development has been studied at various chord-wise locations along the blades surfaces. It is observed that at the effective blowing ratio a single row of cooling injection at approximately $8.25 \%$ of axial chord behind the leading edge may be sufficient to create sustained cooling boundary layer over the entire turbine blade surfaces. The present study has been carried out with cold flows at low speeds to accommodate experimental validation in a low speed cascade tunnel in near future. The heat transfer related issues are not part of the present study.
\end{abstract}

Keywords: Turbine Cooling, Blowing Ratio, Coolant Boundary Layer, Streamline dissipation

\section{Nomenclature:}

BR

CRV

LE, TE

V

$\rho$

Subscripts:

$\mathrm{S}$

$\infty$

\author{
Blowing Ratio \\ Counter Rotating Vortex \\ Leading Edge, Trailing Edge \\ Fluid velocity, $\mathrm{m} / \mathrm{s}$ \\ Fluid density, $\mathrm{kg} / \mathrm{m}^{3}$ \\ Secondary Fluid related \\ Primary flow in the blade passage related
}

\section{Introduction}

In the modern aero gas turbine engines, turbine plays a vital role for its operational efficiency. So to increase thermal efficiency and power output of engine, one of the powerful method is to increase turbine inlet temperature (generally in the range of $1400 \mathrm{~K}-1900 \mathrm{~K}$ ) and operating at such high temperature leads to thermal failure of turbine blade. So effective and sustained cooling is very important for its operational life. There are several different cooling methods that are available for both internal and external cooling [1,2]. But the present work focus mainly on the external cooling technique i.e. film cooling.

In film cooling cold air bleed is tapped from the compressor stages and after bypassing the combustion chamber is delivered to the turbine blade cooling channels. The coolant air discharges through a number of small cooling holes onto the surface of blade which forms a protective thin layer of relatively cold air over the surface and this layer provides protection to the blade against the hot combustion products and also reduces the heat flow from the outside. The bleeding of air from the compressor for the cooling leads to reduction of mass flow of the primary air flow and hence optimum utilization of cooling mass flow is also very important.

The figure-1 shows a diagram of cold air being introduced (normally sourced from the compressor of a gas turbine engine) to turbine blade, where cold air temperature is around $600-800 \mathrm{~K}$, which is hardly a cold air, but it is well below 
the hot gas and the blade operating temperature. The reason for using not-so-cold air is to reduce high thermal gradient in blade, which may lead to structural failure or breakage of blade.

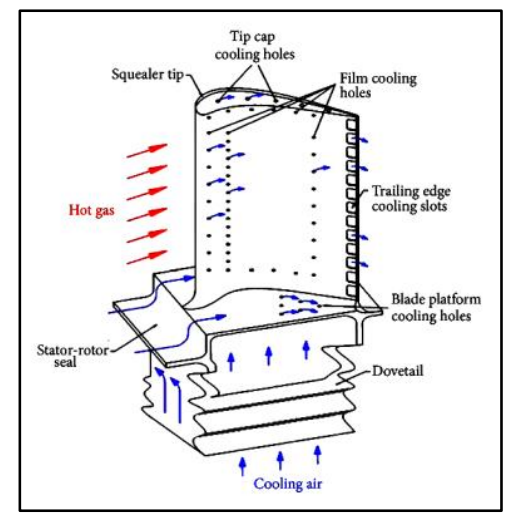

Fig. 1: Typical turbine blade cooling arrangement [9].

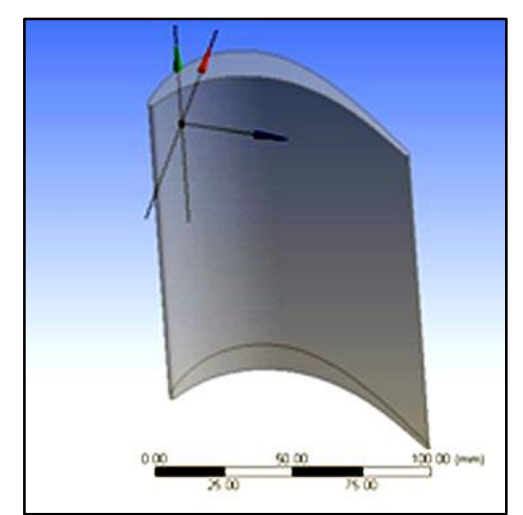

Fig. 2: T-106a turbine blade schematic.

The primary aim of film cooling is to decrease the coefficient of heat transfer from main hot gas flowing through the turbine, to the blade and to increase its effective insulation. In the past several experiment have been conducted to measure those parameters and also to optimize them. One of the parameters that helps quantify the film cooling effectiveness is "blowing ratio". Blowing Ratio is the ratio of mass flux of coolant flow to main flow.

$$
\text { Blowing Ratio }(\mathrm{BR})=\left(\rho_{\mathrm{s}} V_{\mathrm{s}}\right) /\left(\rho_{\infty} V_{\infty}\right)
$$

The present work is only cold flow analysis, so that the density of both the flowing fluids is same; hence, the ratio of the densities is unity and the blowing ratio, therefore, is only the velocity ratio. A CFD analysis with the secondary film with different of blowing ratios in the range of 0.5 to 1.2 and the angle of injection in the range of $25^{\circ}$ to $40^{\circ}$ is carried out using ANSYS CFX ${ }^{\odot} 14.5$ on the T106a (Fig.2) blade in cascade formation to find an effective combination; and also to visualize the aerodynamic behaviour and effectiveness of film formation on the surfaces of the turbine blade.

\section{Past Work}

In the past several experiment have been conducted to measure those parameters and also to optimize them. Han and Ekkad [3] and Rezasoltani et al [4] summarized the past development in blade film cooling with including all the experimental parameter like blowing ratio, angle of injection and effects of wake on cascade blade and also the effect of rotation on the film cooling. Significance and effects of the hole shape and the general ranges of turbine blade parameters are given by Kolghatgi and Acharya [5]. The main influence of coherent structures on the film cooling is identified with LES. and also effect of interaction between main flow and coolant is studied by Rozati et al [6]. 3D flow analysis film cooling on turbine cascade blade for variation of $\mathrm{Cp}$ is studied by Fottner et al [7]. Nemdili et al [8] studied the computational modeling of film cooling considering the vortex generation at the coolant and main flow interaction. In the modern era CFD has become an alternative method to experimental process, which brings down the cost of studies as it accurately predicts the performance behaviour.

\section{Present Work - CFD formulation}

In the present work ANSYS CFX 14.5 is used as CFD tool. The K- $\varepsilon$ model is used for simulation of film cooling and it is a most generally used with SST turbulence model which generally consist of two transport equations. The first transport equation is for turbulent kinetic energy $(\mathrm{k})$ and second equation is for the rate at which turbulent kinetic energy dissipates. This model points mainly to the parameter which affects the turbulent kinetic energy and the assumption made in this model is that the turbulent viscosity is isentropic.

The blade profile and its dimensions are shown in Fig.3. The corresponding CFD domains consisting of the blade, the inlet domain and the exit domain is shown in Fig.4. The secondary flow injection holes are modelled at various chordwise locations inside the blade for preliminary analysis of the secondary flow behaviour as it exits the hole mouth. In this 
report only the first hole injection has been considered for secondary flow behaviour for all analysis. The external flow conditions are shown in table.1. The blade and the cascade geometry details are shown in table.2.

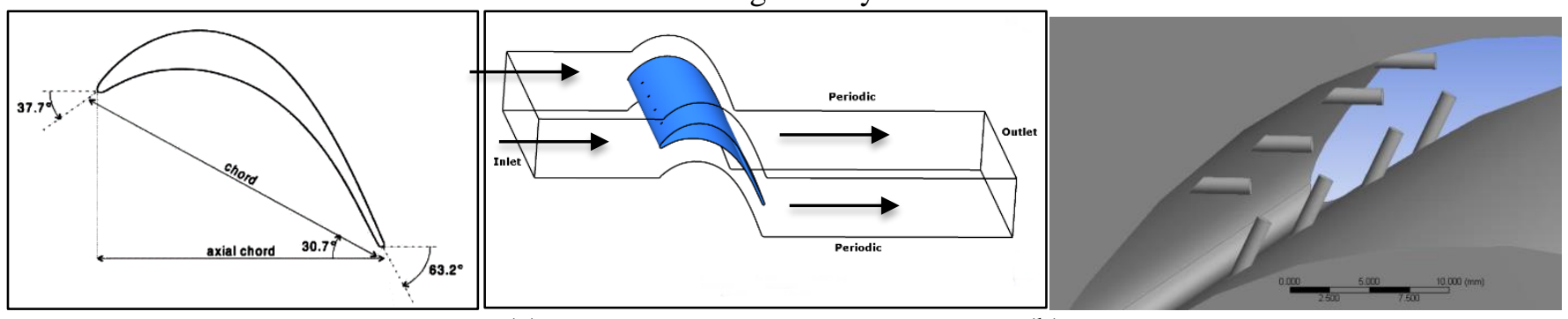

(a)

(b)

Fig. 3: T106a blade dimensions. Fig. 4: Complete CFD domain and its boundaries (a) outside, (b) inside the blade.

Table 1: Flow conditions.

\begin{tabular}{|l|l|}
\hline Parameter & Boundary Conditions \\
\hline Primary inlet velocity & $30 \mathrm{~m} / \mathrm{s}$ \\
Secondary inlet velocity & $24 \mathrm{~m} / \mathrm{s}$ \\
At Wall & No slip condition \\
Outlet static pressure & $101200 \mathrm{kpa}$ \\
\hline
\end{tabular}
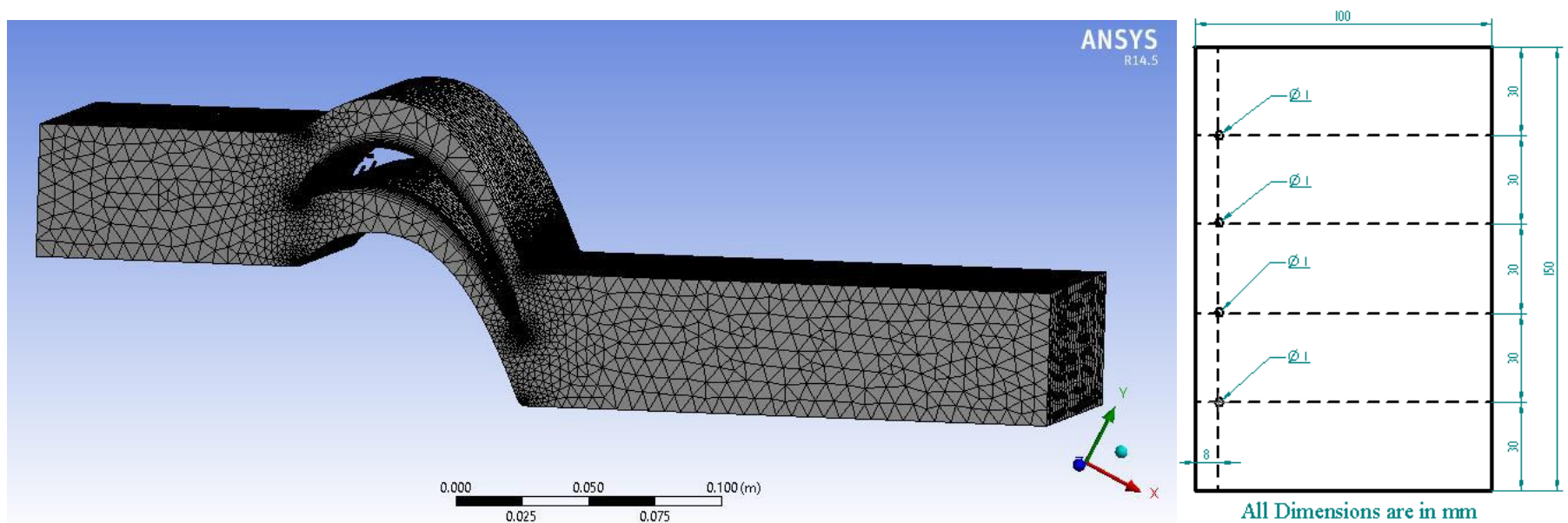

Fig.5(a) Grid around the blade

Fig. 5 (b) Location of the cooling holes

Table 2: Blade and Cascade geometry.

\begin{tabular}{|l|l|}
\hline Parameter & Value \\
\hline Number of Blades & 6 \\
Chord & $100 \mathrm{~mm}$ \\
Pitch & $50 \mathrm{~mm}$ \\
Span & $150 \mathrm{~mm}$ \\
Inlet Flow Angle & $37.7 \mathrm{deg}$ \\
Outlet Flow Angle & $63.2 \mathrm{deg}$ \\
Blade Stagger & $30.7 \mathrm{deg}$ \\
\hline
\end{tabular}

The mesh is generated using ANSYS ${ }^{\odot}$ ICEM, the software package commercially available for creating mesh and it is generated for ANSYS CFX ${ }^{\odot}$ solver. The meshed domain is shown in the Fig.5(a). The mesh generated for flow domain 
is mixed type; it consist both hexahedral and tetrahedral mesh. Hexahedral mesh is created near the vicinity of blade surface to visualize the boundary layer resolution and tetrahedral mesh is created in the remaining space of the flow domain. Mesh generated consist a total elements 2916672 with 861676 nodes. Minimum size of element is $6.2068 \times 10^{-5} \mathrm{~m}$ and maximum size of element is around $1.2054 \times 10^{-2} \mathrm{~m}$. The convergence criteria is taken as RMS residual and factor taken is $1 \mathrm{x}$ e-4 and maximum number of iterations set at 700 .

For film cooling simulation a very important parameter is the blowing ratio. The location of the secondary flow injection is shown in Fig.5(b). The velocity is taken as an inlet boundary condition for both the inlet primary and secondary flows. At the outlet static pressure is applied and at the walls - at the hub and tip ends of the blade periodic boundary condition are applied at both the upper and the lower surfaces. The values taken for boundary condition are based on the iterative process and convergence factor is taken as 1xe-4 for all the cases. Turbulence intensity applied is same for both the main and the coolant flows.

\subsection{Results: Effect of Blowing Ratio}

In the present work inlet velocity $30 \mathrm{~m} / \mathrm{s}$ is taken as constant parameter for all cases and only the secondary flow velocity at the point of ejection is varied to make different blowing ratios - 0.5, 0.6, 0.8, 0.9, 1.0 and 1.2. Different combination of blowing ratios is simulated with different angle of ejection (25,30, 35 and 40 degrees) by keeping all other boundary conditions constant and results for the best angle, $30^{\circ}$ angle of ejection on both surfaces, are presented. The blowing ratio has been applied equally on the suction and the pressure surface.

Table 3: The velocities applied for various blowing ratios.

\begin{tabular}{|c|c|c|c|}
\hline SL No. & Blowing Ratio (BR) & Primary Velocity (V $\infty$ ) & Secondary velocity (Vs) \\
\hline 1 & 0.5 & $30 \mathrm{~m} / \mathrm{s}$ & $15 \mathrm{~m} / \mathrm{s}$ \\
2 & 0.6 & $30 \mathrm{~m} / \mathrm{s}$ & $18 \mathrm{~m} / \mathrm{s}$ \\
3 & 0.8 & $30 \mathrm{~m} / \mathrm{s}$ & $24 \mathrm{~m} / \mathrm{s}$ \\
4 & 0.9 & $30 \mathrm{~m} / \mathrm{s}$ & $27 \mathrm{~m} / \mathrm{s}$ \\
5 & 1.0 & $30 \mathrm{~m} / \mathrm{s}$ & $30 \mathrm{~m} / \mathrm{s}$ \\
6 & 1.2 & $30 \mathrm{~m} / \mathrm{s}$ & $36 \mathrm{~m} / \mathrm{s}$ \\
\hline
\end{tabular}

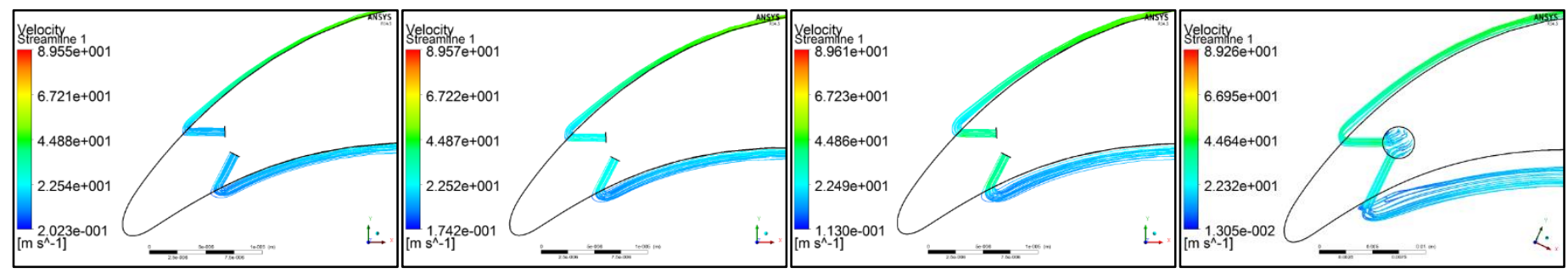

(a) $\mathrm{BR}=0.5$

(b) $\mathrm{BR}=0.8$

(c) $\mathrm{BR}=1.2$

(d) $\mathrm{BR}=1.5$

Fig. 7: Flow on Turbine blade surfaces at various blowing ratios.

Blowing ratio 0.5 at $30^{\circ}$ angle of injection is considered to be the best from preliminary visual analysis, because the coolant flow attaches as soon as it comes out of the hole and the counter rotating vortex pair (fluid mechanically loss making) is weak. Counter rotating vortex (CRV) pair is one of the parameter which affects the film effectiveness and it forms when an injected secondary flow interacts strongly with the primary flow. The behavior of this interaction, if weak, leads to the primary flow to come in contact with the surface, resulting in higher heat transfer in a hot flow situation. It is observed that the intensity of the vortex pair is low at $\mathrm{BR}=0.5$ and is prominent from $\mathrm{BR}=1.2$. At blowing ratio 0.5 secondary flow film completely attaches the surface on both side as soon as it comes out of hole, without forming the vortex, as shown in figure 7(a) and Fig.9. Fig. 7(d) shows strong blowing resulting in the flow shooting in to the main flow in the passage. With closer inspection as shown in figure 8 it is observed that the boundary layer streamline is discontinuous in nature near the trailing edge, which indicates film dissipation, boundary layer transition and development of turbulent boundary layer, which is not conducive to protection from hot primary flow. The CRV pair formation is quite 
prominent at blowing ratio of 1.2 (Fig.9). At the higher blowing ratios formation and propagation of the vortex pair is higher. At higher blowing ratio effectiveness of film cooling is higher due to more availability of cold air on the blade surface. However it is the aim of cooling technologists to reduce the usage of cooling air to minimum, especially in aero engine applications.

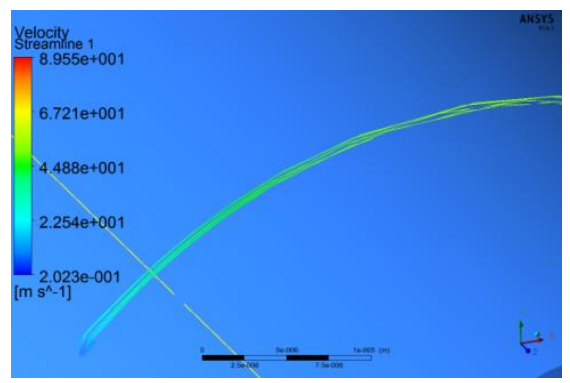

Fig. 8: Boundary layer at $\mathrm{BR}=0.5$.

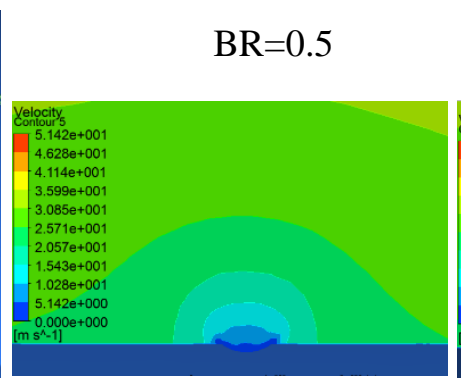

$\mathrm{BR}=0.8$

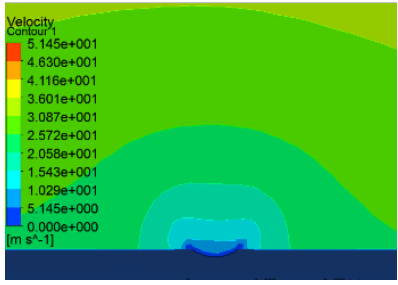

Fig. 9: Near hole flow behaviour at various blowing ratios.

\subsection{Streamline Dissipation with varying blowing ratio}

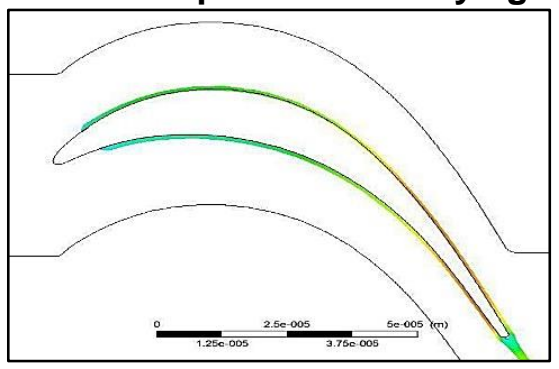

(a) $\mathrm{BR}=0.5$

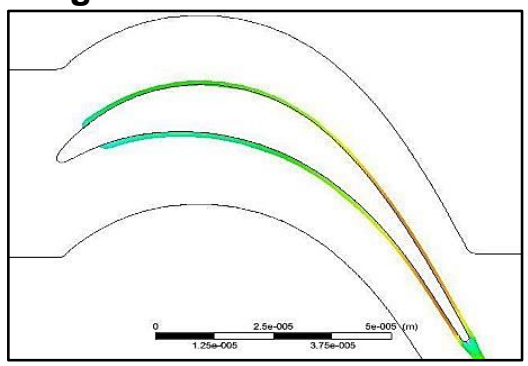

(b) $\mathrm{BR}=0.6$

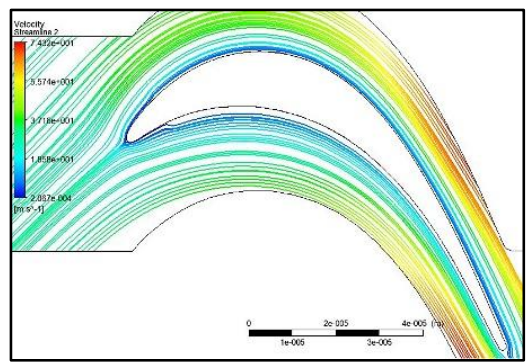

(c) $\mathrm{BR}=0.8$

Fig. 10: Coolant Flow on Turbine blade surfaces at various blowing ratios.

The behaviour of the coolant flow on the blade surface at various blowing ratios is captured in Fig.10. At lower blowing ratios 0.5 and 0.6 it is seen that the boundary layer on both surfaces is accelerated (turn yellow and red colour) and tend to become turbulent, even as they are attached boundary layers. At BR=0.8 the boundary layer remain un-accelerated, laminar and yet attached till the trailing edge. The flow acceleration is observed away from the surfaces. And, as observed before, at higher blowing ratios (BR'1.0) the boundary layer formed by the coolants hold up alright, but at the expense of higher coolant flows. At BR>1.0 the secondary flow shoots in to the main flow in the passage, first on the pressure surface.

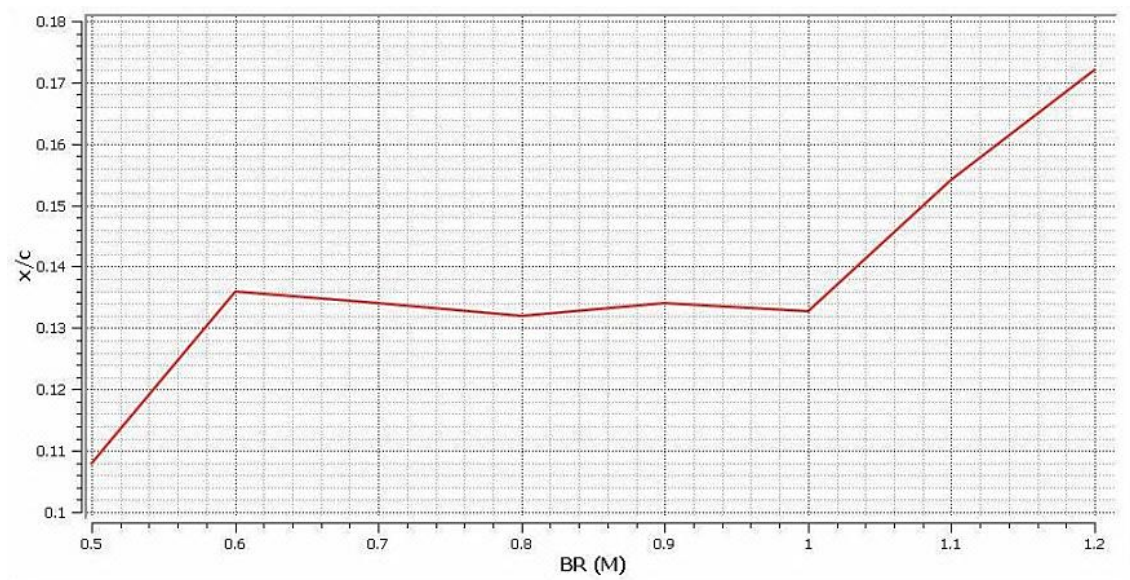

Fig. 11: Coolant boundary layer streamline dissipation with respect to blowing ratio. 
Figure 11 represents the streamline dissipation with respect to the blowing ratio. Streamlines start dissipating due to turbulence or flow separation so at those location new cooling holes has to be introduced for better film cooling performance. According to the plot, the variation of dissipation of streamline is almost constant in the blowing ratio range of 0.6 to 1.0 , whereas below $\mathrm{BR}=0.6$ the dissipation starts much earlier - notably near the ejection hole on the blade surface. At $\mathrm{BR}=0.5$, as per Fig.5, early onset of dissipation leads to early flow transition from laminar to turbulent boundary layer which effectively renders the secondary fluid unfit for cooling purpose in that area. At high BR (>1.0) the higher dissipation distances are high but would entail large coolant mass flows. Fig. 10(c) shows that at BR=0.8 the coolant boundary layer holds till the trailing edge of the blade in spite of showing dissipation tendency much earlier.

On the basis of the above study with varying blowing ratios - it is decided to carry out further studies at a fixed blowing ratio of 0.8 and at the fixed coolant flow ejection angle of 30 degrees. The mass fraction of coolant usage is approximately $0.677 \%$ of the primary air flow around the blade.

\subsection{Coolant film behaviour in 3-D plane of the blade surface}

The study has been carried out for the blades arranged in a cascade with finite blade length (aspect ratio $=1.5)$. This permits a certain degree of three dimensional flow studies. The coolant is injected from equally spaced holes spread along the blade span. The behaviour of the coolant film boundary layer is observed in the figures 12 .
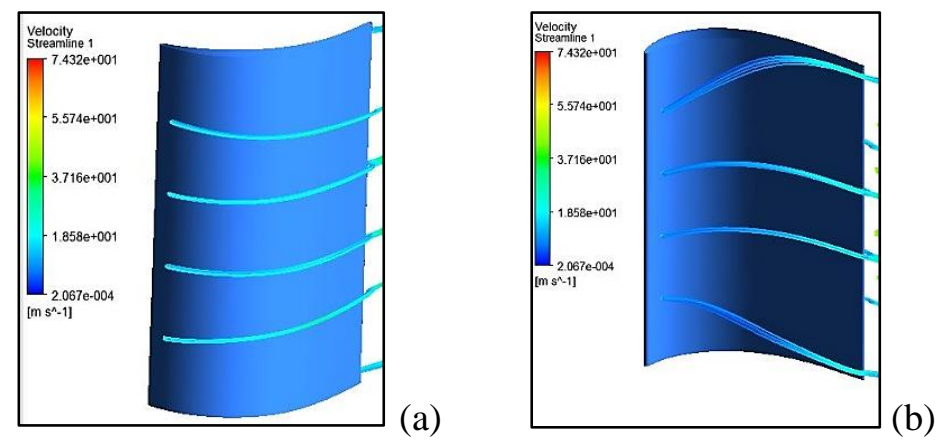

Fig. 12: Film cooling boundary layer spread along the (a) suction and (b) pressure surface at $B R=0.8$.

It is seen that the cooling films, after ejection from the holes, behave differently on the suction and the pressure surfaces. On the suction surface the films come closer to each other, whereas this secondary flow on the pressure surface [Fig.12 (b)] is observed to be dependent on blowing ratio. At $B R=0.8$ the outer two injected films get significantly divergent from the central two rows of films, by the time leave the blade surfaces. Consequently the secondary films leave the blade surface at different span wise positions on the suction and on the pressure surfaces. On a hot flow condition this would significantly affect the cooling effectiveness on the two respective surfaces, and would warrant more cooling holes to be deployed.

The aerodynamic losses incurred by the coolant flow injection has been studied with the help of total pressures computed at the inlet and the exit of the blade. The total pressure along the blade span is shown in Fig.13.
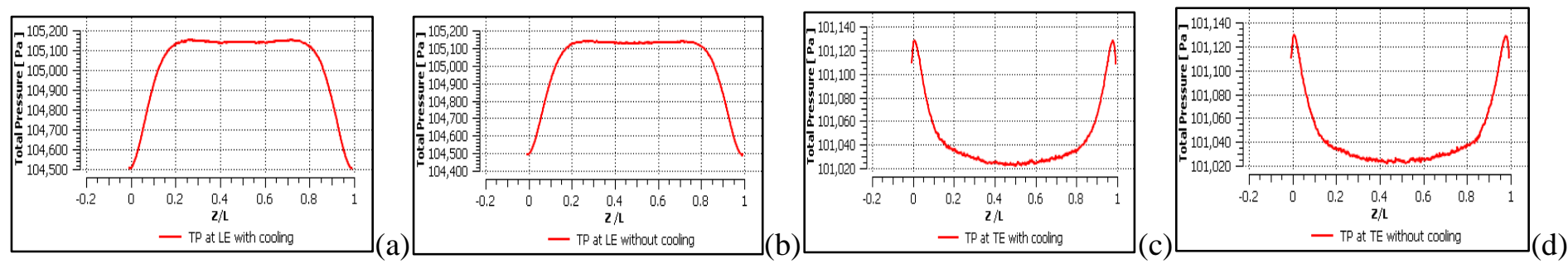

Fig. 13: Spanwise Total Pressure at: (a) LE with cooling; (b) LE w/o cooling; (c) TE with cooling; (d) TE w/o cooling.

The pressure capture shows significant end wall boundary wall development at both the ends of the cascade blades. The pressures at the TE are slightly fluctuating and unsteady, as is normally expected. The effect of cooling is captured in the instantaneous total pressure loss variation plotted in Fig.14. The loss peaks are just behind the coolant streamline. The 
span wise averaged loss is seen to be less than $1 \%$. The steady state averaged total pressure drop across the blade is plotted in Fig.15. Total pressure drop due to coolant (secondary flow) injection is seen to be minimal for blowing ratio 0.8. The total pressure loss (or drop) is the small penalty one has to pay for introducing the coolant film on the blade surface.

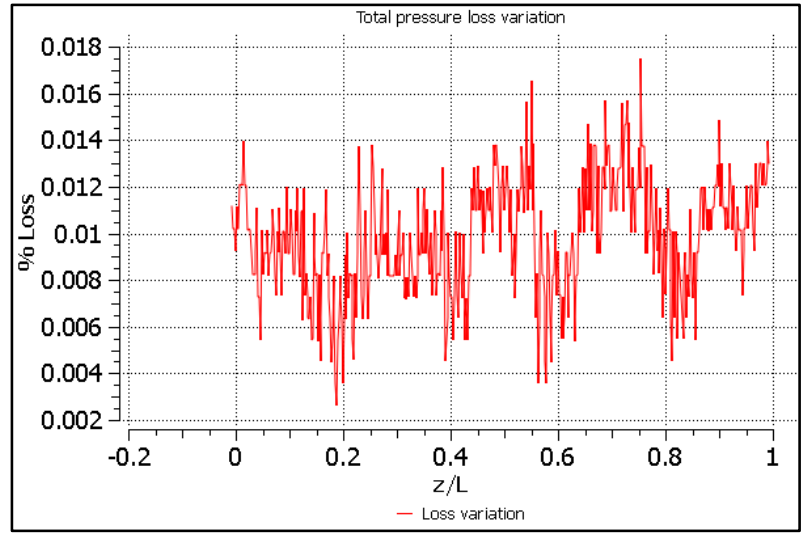

Fig. 14: Total pressure loss variation along blade span.

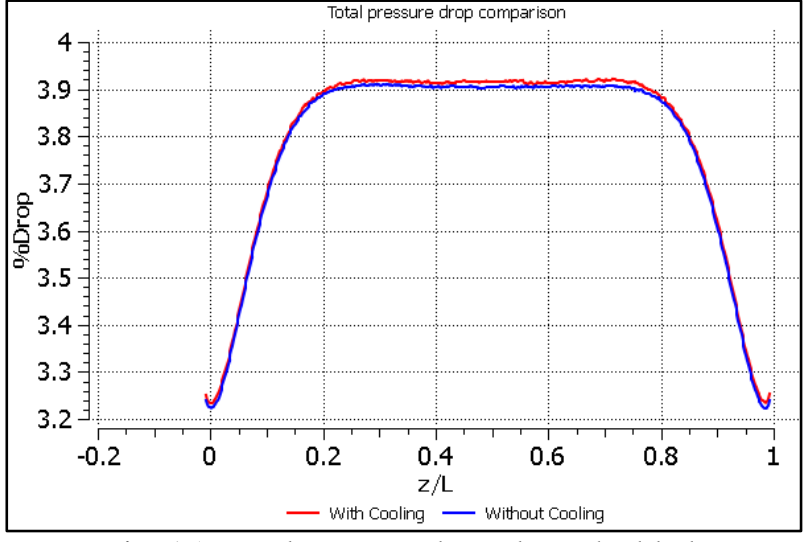

Fig. 15: Total pressure drop along the blade span.

\subsection{Fluid Flow behaviour in the blade passage due to secondary fluid injection}

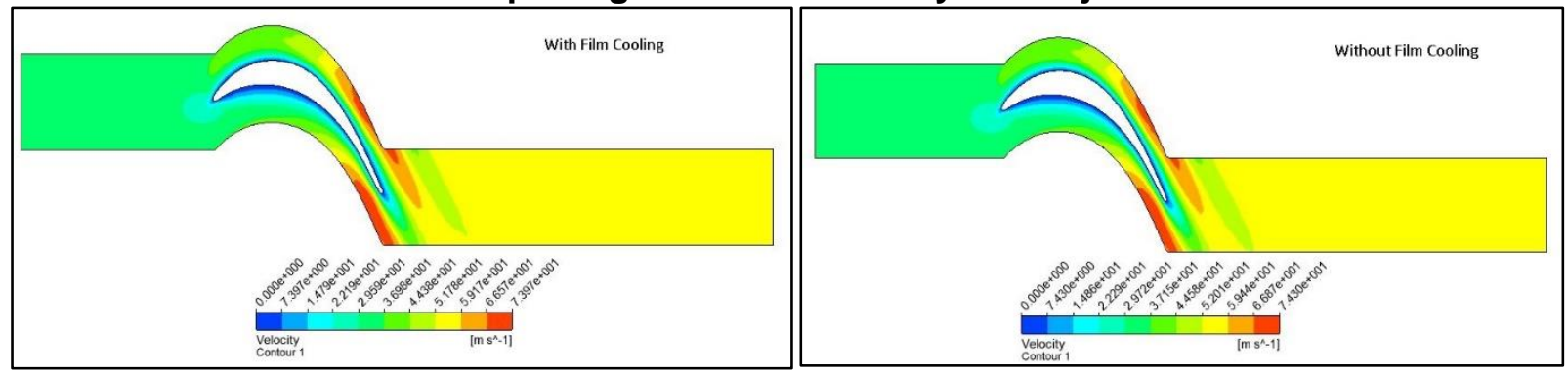

Fig. 16: Velocity contours of flow around the blade profile with and without film cooling flow.
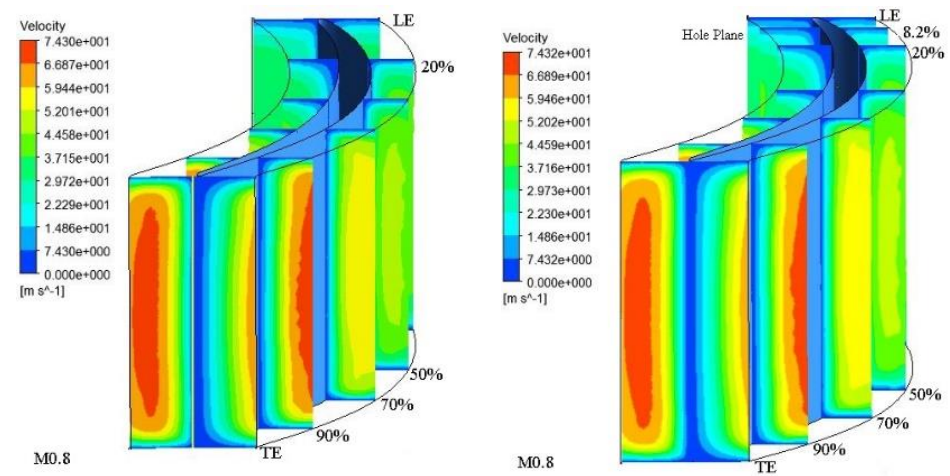

Fig. 17: Velocity contours in the flow passage with and without secondary flow injection.

The flow behaviour through the passage between two subsequent blades, arranged in cascade formation, has been studied for observation of the effect of secondary flow injection of the blade surfaces at a prescribed blowing ratio of 0.8 . The figures 16 and 17 show a very good retention of the basic accelerating flow through the turbine blade passage when secondary flows are applied (for cooling hot blades). This characteristics is important for work extraction capability of the turbine blades.

\section{Conclusion}

The study presented here is intended to show through a CFD analysis that a single secondary fluid injected on to a turbine blade surface at suitable chord wise location could prove to be sufficient for effective cooling of the turbine blades. 
The overall comments borne out of this study may be summarized as follows:

1) An injection location near $10 \%$ behind LE of the blade is seen to be effective. The exact location would vary a little depending on the blade profile and the internal impingement cooling deployment for LE cooling internally.

2) Blowing ratio of around 0.8 should prove to be effective when applied at the chosen location of injection. It is, of course, observed that slightly different blowing ratio may be more accurately determined for the pressure and the suction surfaces. The present configuration shows that $\mathrm{BR}=0.8$ applied at $10 \%$ behind LE on the pressure surface produces secondary flow that slightly intrudes in to the main flow.

3) Blowing ratio much higher than 0.8 shall produce two contra rotating vortices at the hole mouth and, thus is likely to create higher fluid mechanic losses on the blade surface.

4) The secondary flow in a film over the blade surface should not start dissipation for another $10 \%$ of the chord, or till is over the blade "hump". Then it is most likely to sustain its laminar boundary layer characteristics till the trailing edge of the blade and thereby prove to be an effective cooling agent. This is achieved above BR>0.5.

5) The fluid flow interaction between the injected secondary flow and the primary flow over the blade is seen to entail a very small pressure loss penalty $(<0.5 \%$ of total pressure). This is normally acceptable.

6) With application of secondary flow the behaviour of the primary flow in the blade passage undergoes very minor changes. The velocity contours show that the accelerated flow region has become smaller in size. However, mass averaged exit flow velocity remains same, indicating no adverse effect on turbine functioning.

The present study has been carried out with cold flows at low speeds $(\mathrm{M}<0.1)$ to accommodate the option of experimental study of the same coolant flow behaviour in a low speed cascade tunnel - to be carried out in near future. The heat transfer studies and cooling measurements are not intended to be part of the study.

\section{References}

[1] A. F. El-Sayed, Aircraft propulsion and Gas turbine engines, CRC Press, 2008.

[2] P. Hill and C. Peterson, Mechanics and Thermodynamics of Propulsion, 2nd Ed. Addison Wesley, 1992.

[3] J. C. Han and S. Ekkad, "Recent Development in turbine blade film cooling," International journal of rotating machinery, vol. 7, no. 1, pp. 21-40, 2001.

[4] M. Rezasoltani, K. Lu, M. T. Schobeiri, and J. C. Han, "A combined experimental and numerical study of the turbine blade tip film cooling effectiveness under rotating condition," in ASME Turbo-Expo Conference, GT2014-25625, 2014.

[5] P. Kalghatgi and S. Acharya, "Improved film cooling effectiveness with a round film cooling hole embedded in a contoured crater," in ASME Turbo-Expo conference, GT2014-26600, 2014.

[5] A. Rozati and D. K. Tafti, "Effect of coolant-mainstream blowing ratio on leading edge film cooling flow and heat transfer-LES investigation," International Journal of Heat and Fluid flow, vol. 29, pp. 857-873, 2008.

[6] D. Bohn, V. Becker, and K. Kusterer, "3D flow analysis of turbine blade cascade with leading edge ejection," Journal of Propulsion and Power, ASME, vol. 16, no.1, pp. 49-56, 2000.

[7] F. Nemdili, A. Azzi, G. Theodoridis, and B. A. Jubran, "RST modelling of film cooling at LE of a symmetrical turbine blade," Journal of Heat Transfer, vol. 29, no. 11, pp. 950-960, 2008.

[8] K. Takeishi, K. Tsukagoshi, S. Aoki, and T. Sato, "Film cooling on a gas turbine rotor blade," Journal of American Society of Mechanical Engineers, Turbomachinery Journal, vol. 114, pp. 828-834, 1992.

[9] A. M. Elsayed, F. M. Owis, and M. M. A Rahman, "Film Cooling Optimization Using Numerical Computation of the Compressible Viscous Flow Equations and Simplex Algorithm," International Journal of Aerospace Engineering, 2013. 